hope that some worker favourably situated for this purpose will succeed in doing so.

Department of Parasitology,

S. AdLer

Hebrew University, Jerusalem.

\section{Determination of Plant Densities}

Is surveying plant associations, it is usual to determine the valence by noting the presence or absence of each species in sample areas, and the density by counting the number of individuals of each species in the same, or other, sample areas. Two difficulties are frequently encountered in determining densities by this method, the length of time needed in the field and the intermingling of root systems of individual plants of the same species with consequent indecision as to the exact number present in the sample. The following method has the advantage of requiring one set of observations only, from which valence and density may be determined, and of avoiding counts within the sample areas.

Suppose the species has a density $N / A$ plants per unit area and that a sampling area $a$ is taken $t$ times at random. Then the expected number of plants per trial will be $a N / A$, and the number $y_{0}$ of trials containing no individuals of the species would be expected to be given by the expression

$$
\begin{gathered}
y_{0}=t \exp (-a N / A) . \\
\text { Whence } \frac{N}{A}=\frac{1}{a} \cdot \log _{e} \cdot \frac{t}{y_{0}} .
\end{gathered}
$$

If the number of trials is 50 and the sampling area 0.1 square metre, a graph relating density and $y_{0}$ can be drawn and shows a remarkable sensitivity for densities 33 per sq.m. to 3 per sq.m. By increasing the sampling area to 1 sq.m., the density values on the graph are reduced ten times, and we have a sensitive range from 3.3 per sq.m. to 0.3 per sq.m.

Much time has been saved in the field and results are reasonably in accord with densities found by the more laborious method.

Biology Department,

E. J. MACHIN

Cheltenham Grammar School. June 26.

\section{Metabolism of $\alpha: \beta$-Dihydroxy- $\gamma-(2-$ methyl- phenoxy)propane ('Myanesin')}

IN a recent communication ${ }^{1}$, Wyngaarden, Woods and Seevers have shown that 'Myanesin' administered intravenously to dogs appears in the urine mainly in combination with glycuronic acid, while a small amount is excreted without change. We have found that after intravenous administration to humans or rabbits, a portion is oxidized and excreted as $\alpha$-hydroxy- $\beta-(2$-methylphenoxy $)$ propionic acid. The acid may be isolated by acidifying the urine to $p H$, extracting with ether continuously for 24-48 hours, transferring from the ether extract into sodium bicarbonate solution and recovering from the aqueous solution by acidifying and extracting with ether. The ether-soluble product is crystallized first from carbon tetrachloride, then from water and finally from chloroform. The pure acid melts at $146-147^{\circ}$ (corr.) (found: $\mathrm{C}, 61 \cdot 2 ; \mathrm{H}, 6 \cdot 1 ; \mathrm{C}_{10} \mathrm{H}_{12} \mathrm{O}_{4}$ requires $\mathrm{C}, 61 \cdot 2$;
H, 6.1 per cent). The acid is not optically active. It is identical with the acid obtained by condensing $o$-cresol with $\beta$-chlorolactic acid (found: C, 61.1 ; $\mathrm{H}, 6 \cdot 1$ per cent).

We are indebted to Dr. F. Barnett Mallinson for supplies of urine from patients who had received 'Myanesin'. Our thanks are also due to the directors of the British Drug Houses, Ltd., for permission to publish this note.

Research Department,

E. L. Graves

T. J. ELLiotT

W. Bradley

The British Drug Houses, Ltd., London, N.1. April 15.

${ }^{1}$ Proc. Soc. Exp. Biol. and Med., 66, 256 (1947).

\section{Histamine - Heparin - Thrombin Chain Mechanism}

EARLIER experiments of ours have shown that the organism regulates the coagulability of the blood by the inactivation of thrombin ${ }^{1,2}$. Many factors take part in the system causing the thrombin to disappear. We distinguish an adsorption effect and a fermentative inactivation process, and indicate the inactiv. ating process by its reaction velocity factor.

We have worked out a simple and rapid process for investigating the inactivation ${ }^{3}$. We put thrombin of a known activity into the serum under investigation, and after an incubation period of $1,2,3,5$ or $10 \mathrm{~min}$., we determine the time in which samples taken from the mixture make the fibrinogen coagulate. As a result of the inactivation we get increasingly longer clotting-times. From the clotting-times we determine their thrombin activity on the basis of an empirical relation, and from the data thus obtained we calculate the reaction velocity factor $(k=1 / t \log$. nat. $c o / c)$.

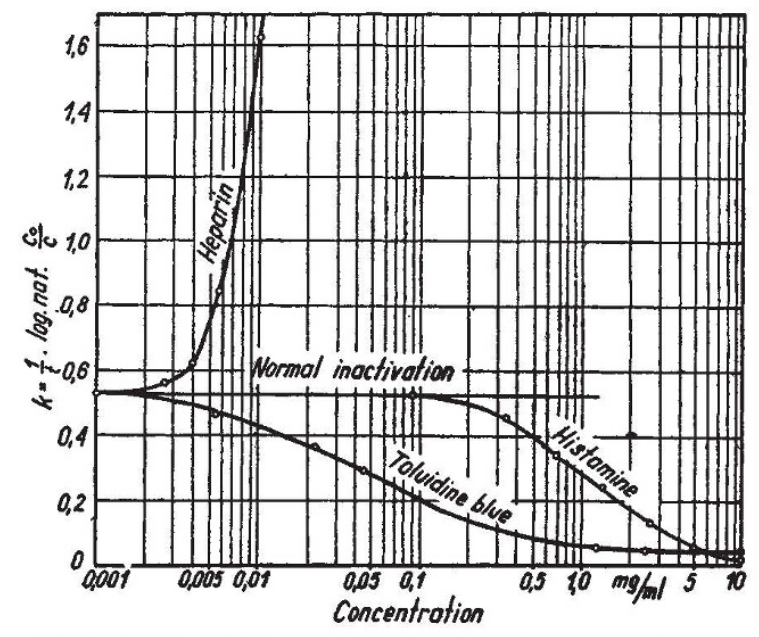

Change in thrombin inactivation reaction-velocity constant under the effect of heparin, toluidine blue and histamine respectively, in rabbit serum

Heparin increases the reaction velocity of the inactivation (see graph). If we bind the heparin with toluidine blue, the velocity of the inactivation diminishes decidedly ${ }^{4}$. We find that histamine, both in vitro and in vivo, likewise strongly decreases the 